\title{
L'oeuvre scientifique de l'expédition de la «Belgica»
}

In: Annales de Géographie. 1905, t. 14, n74. pp. 170-176.

Citer ce document / Cite this document :

Zimmermann Maurice. L'oeuvre scientifique de l'expédition de la «Belgica». In: Annales de Géographie. 1905, t. 14, n74. pp. 170-176.

doi : $10.3406 /$ geo.1905.6471

http://www.persee.fr/web/revues/home/prescript/article/geo_0003-4010_1905_num_14_74_6471 
lemploi de la main-dixurre indigène! dorot-on que las indigènes nobaient pas aussi aptes que les ltaliens à creuser les formes de radoub de l'arsenal

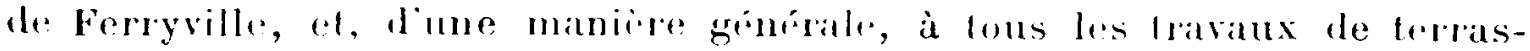
sement? Croit-on qu'on ne purratit les utiliser plus quon ne le fait dans les mines et dans les rarribes, en faire des gardes-barribes et des cantonniers? A Potimsille et dans d'autres vignobles, n'emploie-ton pas un personnel exclusirement indigine? S'il n'est pas facile d'imposer aux entrepreneurs dr travaux publies lemploi d'une forte proportion douvriers fancais, me peut-on, du moins, exiger quils sadressent aux indi-

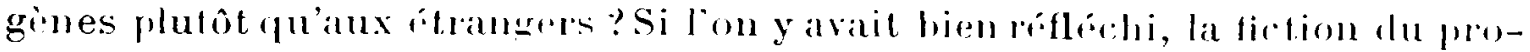
fectorat, dont on s'est parfois servi pour entraver le peuplement francais,

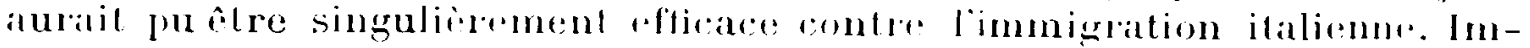
puissants à peupler l'Alrirur du Nord dhommes de notre sang, la presente. done population indigine nombreuse ef tenace, si elle est une cause de dif-

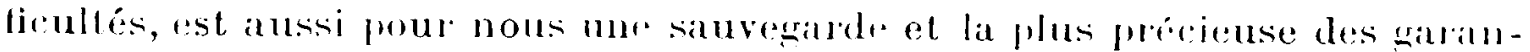

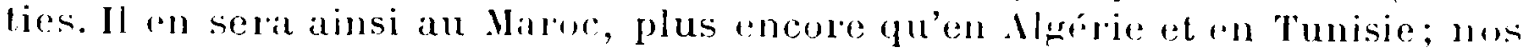
intérits sont dacrord arec reux des musulmans pour eviter dy rien brusquer, pour conserver les rouages actuels dans la mesure du possible el pour preferer, en loule cireonstance, la solulion indigrine is la solution internationale.

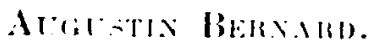

\section{LONITRE SCIENTIFIQUE DE L'EXPEDITION DE LA " BELGICA "}

Depuis les articles déjàtanciens ${ }^{1}$ oì nous arons exposé quelques-uns des resultats d'ensemble recueillis par l'expédition antarctique belge de $\mathrm{M}^{\mathrm{r}} \mathrm{w}$. lierlache, la publication des résultats délinitifs dans une série de rapports scientitiques a fait d'importants progries. C'est le gouvernement belge luimòme qui s’est chargé d'éditer ces rapports à ses frais; la commission de la "Belgica ", présidée à l'origine par le général Barabonr, el complant parmi ses membres, MIrs de likrlache, vice-président; $i$. I.fconte, secrílaire;

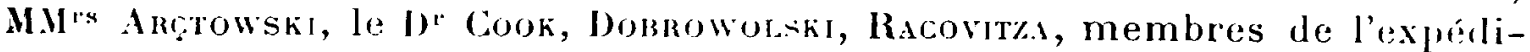
tion, et une série de savants belges, a blé chargée de surveiller la mise en ceuvre el la publication des nombreux matériaux recueillis. Elle s'est asisuré, sur la proposition des membres du personnel scientitidue de la mission, le concours de ruatro-vingts collaboratents, rhoisis parmi les sants du monde entier, et au nombre desquels tigurent une vingtaine de Frangais :- liensemble des rapports formera un ouvalog luxueuxet de grandes proportions: on ne privoit pas moins de dix volumes qui seront consacrés allx mationes

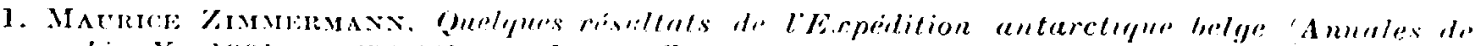

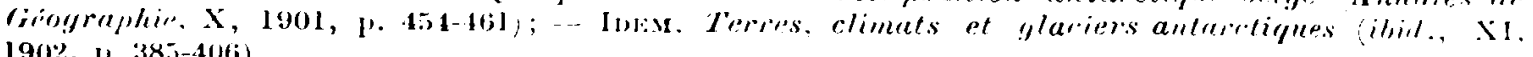
$1902,1 . .38 \%-40(6)$.

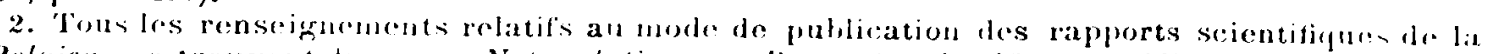

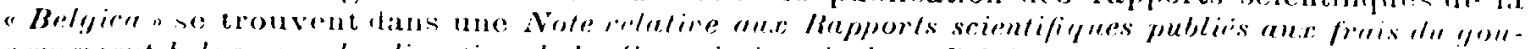

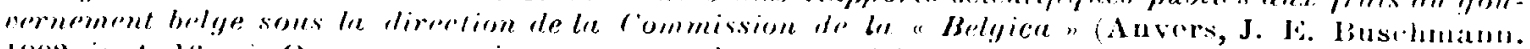
1902, in-1, 16 p j. On y pent voir notamment la composition le la commission de la "Belyire", la

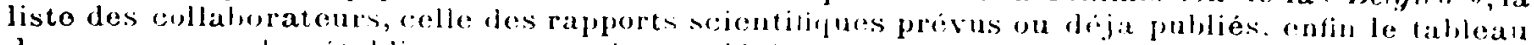

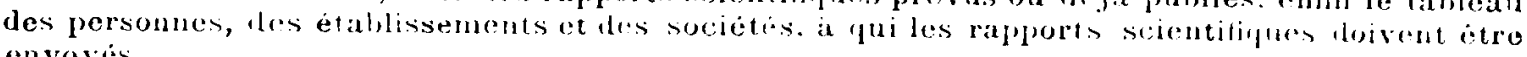
envoyis. 
suirantes: 1. I, Relation du voyage et resmme des resultals; travalux hydromraphiques et instruclions nautiques; t. II, Astronomie et Physique du gloles:

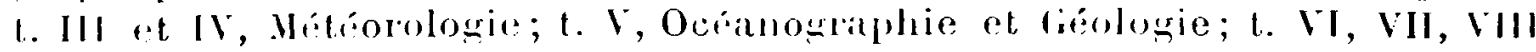
.I IX, Botanique et Zoologre; 1. X, Rapport médical du l) Cook et rtudes anthropologiques sur les Indirms onas'.

Nous avons déjà rendu compte de deux des llémoiles publiés, ceux de

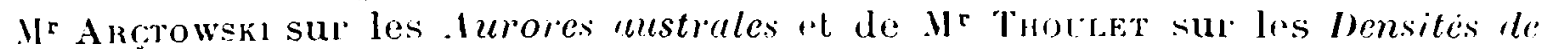
leale de mer. Un srand nombre d'autres rapports ont paru au cours dis années 1902-1904. Voici la liste de ceux que nous avons entre les mains .t qui, par leur dimension sourent considérable, leur esprit minutieusement seientilique, nous donment une idér de plus en plus hatule de la valeur des observateurs de la "Belyica ". Il est i noter en offet quo les plus étendus

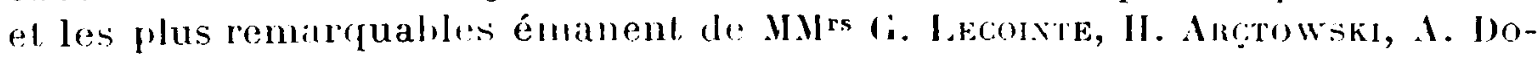

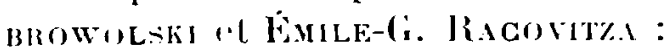

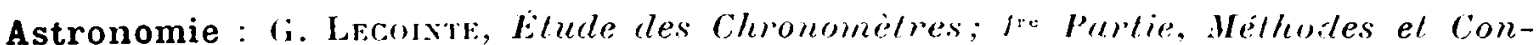

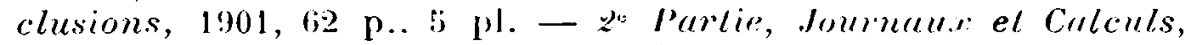
1901. $1: 32$ p.. 1 pl. - les deux parties, $1: 3$ fr. :30.

Météorologie : II. Ancowwski, Plünomènes olliques de l'almosphère. Journal ales

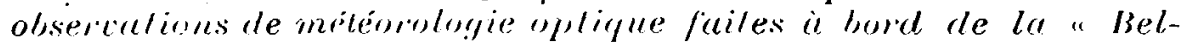

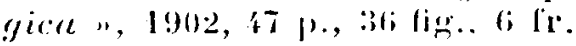

H. Alserowsing, Rapport sue les observalions méteorologiques ho

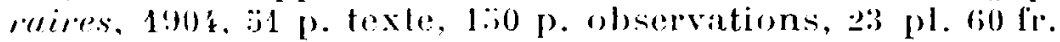

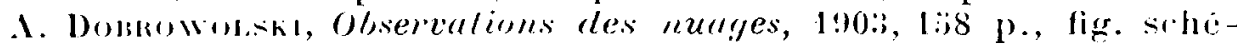
mats. 20 fir.

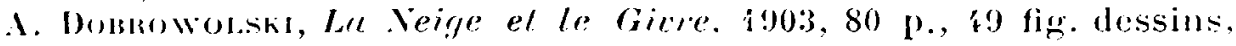
$10 \mathrm{li}$.

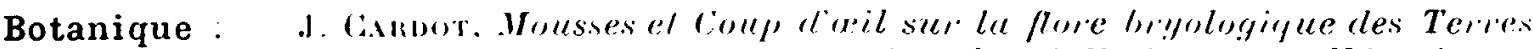

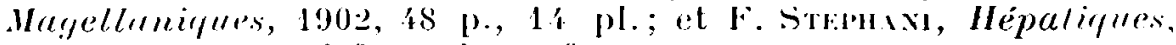
(i p. - lin un seul liase. de ess lr.

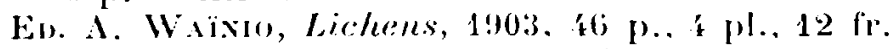

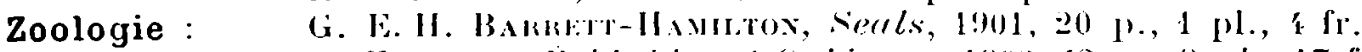

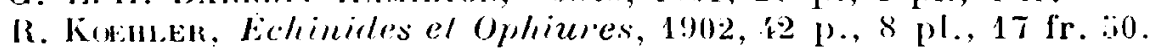

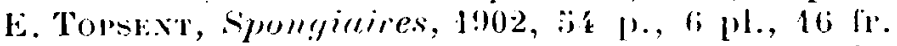

L. Jotisix, Biochiopodes, 1902, 1 t pl., 2 pl.. $\vdots$ fr.

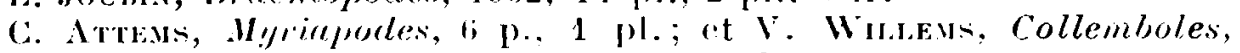
20 p., pl., 190.2. - En un seul facc. de $11 \mathrm{fr}$.

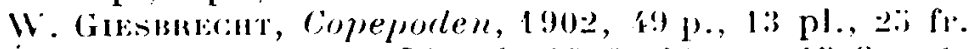

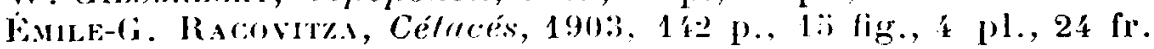

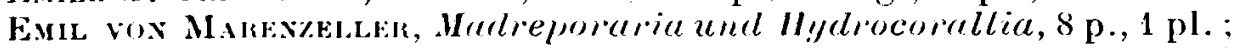

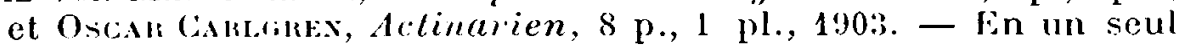
lasc. le ; ipe.

Paul Pelsexish, Mollusques Amplineures, Gastropodes el Lamellih.anclces), sti p., 9 pl.; et L. Jocmix, Gépllalopodes, 2 p., 190:. Fin un seul fasc. de $20 \mathrm{fr}$.

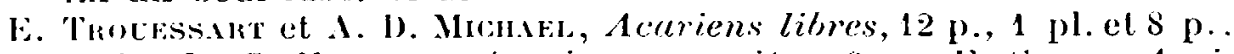
1 pl.: L. G. Necmax, Aconiens prorsiles, 6 p.; E. Sumox, Arai grues el Faucheurs, 8 p., 1903. - En un seul fasc. de 7 fr. 30.

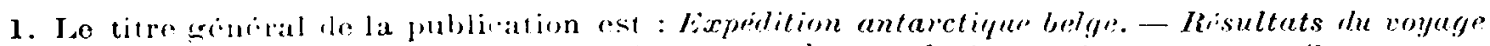

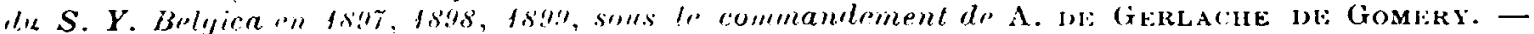

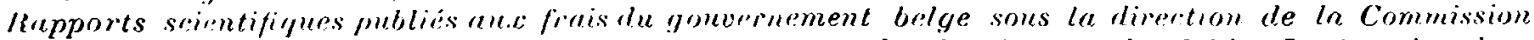
d. La Belfica, Anvers, J.-li. Buschmann, Rempart de la Porte du Rhin. In-4, planches nombreuses. Les volumes I ot $V$ sont tirés a 550 exemplaires, les autres volumes a $50 n$-eulement. Il ne sera mis en ronte que cinquante collections completes des Mimoires. Ils penvent itre acquis, séparément on par séries completes; a Anvers, chez J.-L. Buschmann; a paris, wez Io Soutier: a lierlin, chr\% Friedlander : a Londres, chez bulau \& Co.

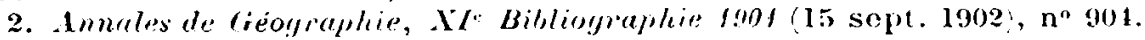




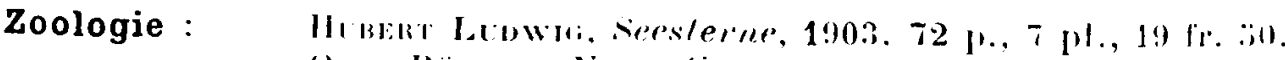

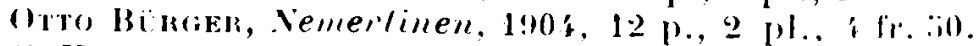

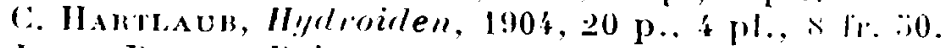

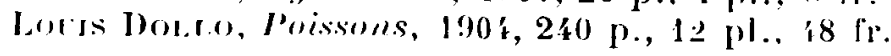

Wapres la scule finumépation des memoires ci-dessus, on se rend compre que crtte collection offre braucoup plus linterit pour les naturalistes qur pour les géosraphes. I a plus grande partir des rapports sur la hestanique el la zoulugie sont remplis par la description des especes, dont les planchres, souvent mervillrusement dessineres (voir notammont les admirables des-

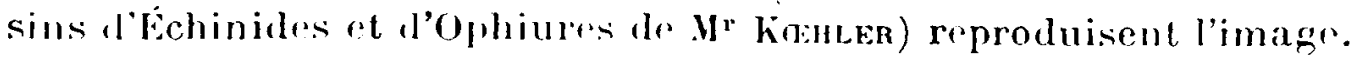

Pomlant il estrare qu'une conclusion gengraphique, si comrtr qu'elle soil, n" se dergage pas dre ces minutieuses monographies. Nous allons insister sur les observations qui nous paraissent offrir, à notre point le vue spécial, l." plus d'intript.

Lidude appofondir que Mr Leconte a faite des chronomètres et la jublication integrale de ses ralduls siexplique aisément quand on mesurelingrortance de cet élément pour la localisalion exacte des sondages, pour la delermination des points servant de reper aux leves hydrographicfues, ef pour létuele les déplacements du navire durant sa délive lans les glaces. Wr Leconte, lui aussi, note linsuftisanee, du matipiel dinbservation de la "Belgica ". La mission ne disposait que de trois rhronomètres, dont un était vieux et usé. Il en 'ût fallu six, solon lui, plus trois montres de torpilleur, deux rompteurs de marine, et trois veritahles chromomitres de porhe. Si la "Belyica " avait ele ciasée par les glaces comme l. fut ensuite l" "Antarctic ", la mission, romme le dimontre $M^{*}$ Lecorve, ent risqué de se tronver dans l'incapaciti de faire aucune observation valable. En outre, les montres n'avaient pas été suffisamment atudiées avant le départ d'Europr. De tout cela, il n'esl, en fait, rien résulté de ficheux. Mais Mr I.rcolste $y$ insiste avec raison, afin que les explorateurs soient lien pénitiris de la nécessitri de possider un matériel scientifique considémble.

Le momoire le $\mathbf{M}^{\mathrm{r}}$ Ancrowsk sur les observations météorologiques horaires est un des plus importants de la serie. Ici les instruments d'observation étairnt excellents, bien que quelques-uns ne fussont quen frartie utilisables dans les regions antarctiques. On regretta notamment de ne pas posséder quelques lhermomètres à minima pour les basses températures. Les observations unt dé faites sur la passerelle de la "Belgica ", toutes les heures, excepté au moment des travaux entrepris pour léliver l. navire. Elles ont éte minuticuses of comportent, dans les tableaux, un grand monbre de notations variées. I.es résultats géniraux ne changent pas grandichose aux chiffes que les notes povisolles de Mr Angowsk nous avairnt fait connaître: seules les moyennes mensuelles et surtout les roses des vents ont subi des molifications notables. Cependant le tableau des températures, publie dans notre articlo ', reste presque identique. Io maximum extreme, pour lequel Mr Angrowsi avait donne le chiffre douteux $+3^{\circ}$, est définitivement lixi $i+2^{\circ}, \because$. Si lon considere l'onsemble des observations, les vents du $S$ ont áté les plus froids (moy. - 160,.i ol les vents du NE. les

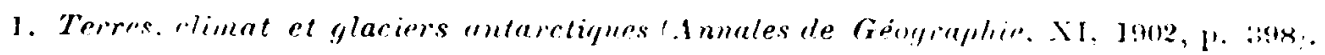




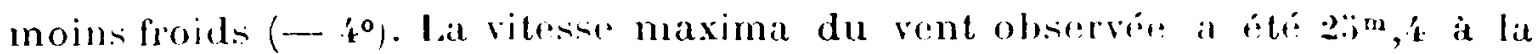
seronde le: 103 septembre; les vitesses de $1:$ a $200 \mathrm{~m}$. ont ele fréquentes dans l.s nuragans en toute saison. Lal nébulosité a été beaucoup plus forte en clé qu'n hiver (un seul jour peu nuageux en févier); juillet a eté relativement. lr. mois le plus clair : la mer grelée se comportait en effel comme une plaine couverte de neige. Il y a eu 260 jours de neige.

Létude des pibinomènes optiques de latmosphere est desplus utiles dans les régions polaires, où le mode d'incidence de la lumière engendre lant de: jeux de couleur flanges el merrolleusement varies. Très curieuses nous paraissent donc les notations de $\mathrm{M}^{\mathrm{r}}$ LreTowsi sur les déformations apparentes des astres à lhorizon (exomple : le soleil prenint la forme d'un pul. muni d'un couvercle, d'un trapieze, d'un rectangle), sur les phénoménes de: mirage, et surtout sur les etonnants phinomines crepusculaires qui carartirisent les rigions sountises à la nuit el aujuur continus. Il y a là un domaine oì l'observation precise ne fait guere que rommencer ${ }^{1}$. Nous ron dirons autant de l'ilude des systemes de nuages a laquelle .l" Dobnowolsk sest rour

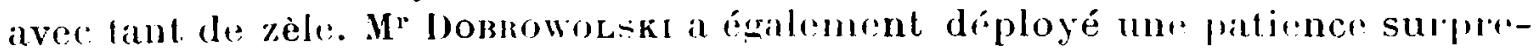
nante dans le dessin des cristaux indétiniment variés de la neige el du grive. L'un des points les plus rurieux qu'il melle en lumich est la variation de lorme et de dimension des cristaux de la neige aver liabaistoment des lempéatures; il contirme à ce sujet les observations de li. Itersuxs, qui arait déjà calculé que la quantite de rapeur dimimunt aveo lahaissement de la tempéralure, les dimensions de certains cristaux (ŕtoiles sans champ central) diminuent lois nellement ares lil tempriature et it peu près dans les mêmes proportions que la quantité de vapenr. Ainsi, alors que la neige

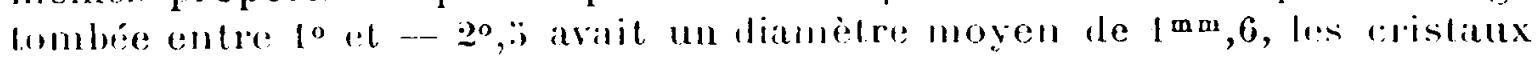

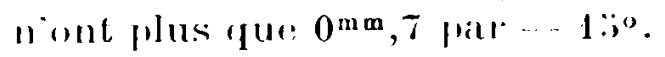

l.es géggraphes s'intéressiront sulout aux conclusions souvent très meuves et inattendues qui ressortent des éludes bolaniques et zoologiques. C'est en examinant dans le détail les nombreuses monographies énumérées plus haut qu'on se rend compte de l'etendue de notre ignorance sur la zone antareti.pue en 1897. Dinns le domaine de la vie, presque tout restait encore a faire. On ne connaissail pas de plante phanérogane au delà de $63^{\circ} \mathrm{S}$, "les demien's virélaux cellulaires observés dans la direction du pôle austral consistaient chl quinze especes d'algues et trois mousses, recueillies par J. D. Hooktir sur l'ile Cockburn, par 6'ro1: $\mathrm{S}$. Ces représentants de la flor: antarcliche étaient si chétifs que Hooker pouvait dire avec raison que cet ilot semblait. itre l" "Ilima Thule " de la régétation australe" ". Or, la mission de la "Belyica" a prouvé lia prenière que les terres antarctiques ne sont pas aussi dépourvues de vie organirque qu'on se l'imaginait. Les découvertes de Mr Racovit\% constiluent ì cet égard une véritable révélation. D'abord il it retrouvé plus loin ver's lo: S. l’Aira antarctira, cette graminée déjà signalće; mais surtout il a rerueilli, dans les anfractuosités à l'abri de la neige et res flots, de florissantes colonies de Housses, presque toujours formées d'associaltions de plusicus espires. ru les plus robustes protigent les plus frèles.

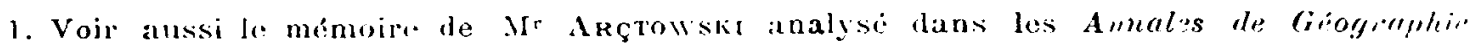

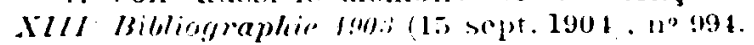

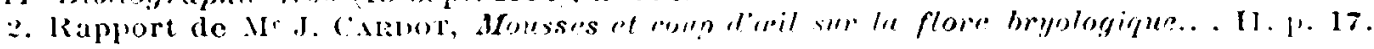


Il est rare de trouver un garon compose dune scule espice. Mr Cinnor distingue vingt-sepl tepices de Mousses actuellement commues, lont quinze especes et deux variétés nouvelles. Pour les Lirhens, Mr hiscovro en a lamassé, sur les cotes du détroit de Gerlache, cinquante-cinq esprices, dont vingt-neul sont nouvelles ou endemiques. Ces lichens antareliques montrent une evolution et une difripenciation tries avancies'.

Lat mème abondance de découvertes a étr problisés en ronlogir. lat "Belgich" penvait se foliciter d'avoir un naturaliste distingué, forthien preparé it sa tarhe, ol dont fr: nom restera disormais, tant par la valeur de ses coludes persomnelles que par la masse de materiaux nouraux quil a rapporlés, Mr Eume-ti. Rocorros. Sans entrer dans le détail de ses dénuvertes, nour voudrions sutul insister sur lévolution quelles ont amenre dans cerlaines idees grougraphiques rourantes.

liune des plus frappantes conclusions qüimpose lexanen de tant de filits nouveaux, cest la diffirenciation de l'immense zone hiologicpue antaretipue, que faute drabments connus, on faisait rontiner directement a la zone tropicale, ef dont Mrbare, OrTmaxx et Trovksant arotaient les limites suivant des lignes variables, mais roisines de $30^{\circ}$ de latilude. On est contraint par l'etude de toutes les formes de la vie animale nu végetale a dis-

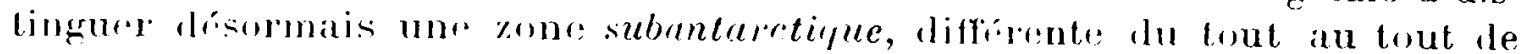
la zonr antaretique, el qui ne fait en somme que correspondre a la rone temprifer des meterorologistes. Tous les savants appelís it se flrononcer sur les materiaux de la "Belgica" insistent indistinctenent sur ce point. Deux exemples au hasard : on comnaissiat seime especes de coprepodes entre a.:" lat. S. et le cerrele polate; on n'en connaissait pas une ì l'intririeur du

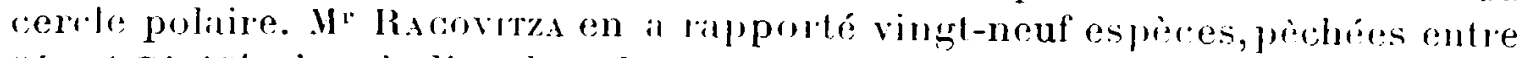
6.'o et $71^{\circ} 18^{\prime}$, c'est-itdire dans la zone antaretigue proprement dite. Sur ce uombre, il $y$ en a dix-lıuit a dix-neul de pélasiques. Le manque de concoldance ext frappant avec les especes anterieurement connues rel cités plus haut, il n'y an a que deux de communes. De mème, ll KoEHLer remarque que les Éhinides subantarrliques different considerablement des rinq espices nouvlles rivélées par llr liacovtas.

Ln autre point capital est celui de l'influence qu'ont eue les découvertes de la "Belgica" sur la théorie aujourd'hui bien ronnue de la bipolarite, soutenue brillamment par Prelien et Murray frappé des ressemblances que lui paraissaient présenter nombre de formes connues dins les drux régions polaires, mais absentes des régions tropicales; il tâchait de l'expliquer par la persistance d'une lame antérieure, qui serait anjourd'hui refoulée aux deux pôles et dans les rigions abysales de l'ocrian. 1) fijà, arant les récentes trouvailles antaretiques, la bipolerite avait souffer de ce fait qu'un grand nombre d'expeces pretendues bipolaires avaint été reconnues simplement cosmopolites. Il semble bien qu'un coup brancoup plus sensible encore lui soil perté allojoulhui. lans les dives domaines de la zoologie antarcetique, tous les savante de la " Belgica "sont frappes du

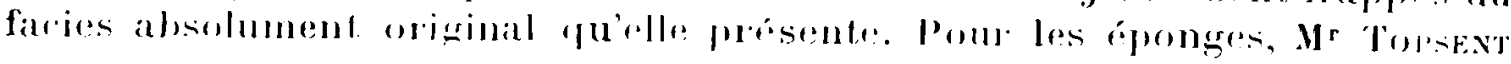

i. A. Wainu, Lir/mens. P. 3.

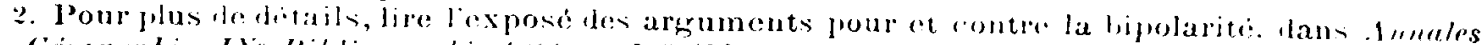

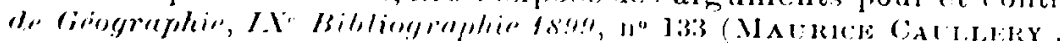


fail remarguer qu'il nexiste qu" trois esperes communes aux faunes des deux regions phlaires et le rosmopulitisme en est d'alleurs arere; il y a, par contre, des genres reprisentés aved une extreme abondance dans les

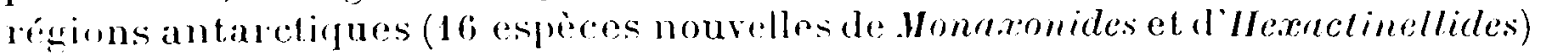
et qui manquent a peu pres completement dans le monde aretique. M" Kanten aftirm. que les Echinides et les ophiures antarcticues ne prisentent pas la moindre analogie avec les formes arclifues et ne permetlent de tenter aucun rapprochement. C'est presque partout la meine uote, bien que pour les Mousses, Mr C.spore reconnaisse d'elroites aftinitís entre les espéces nouvelles découvertes par Mr Racovitza et les espèces boréales. Dans l'ensemble, il semble bien qu'il faille renoncer à la bipolariti dans la forme du moins où la théorie a eté soutenue. Nous indiguons particulièrement la discussion approfondie que consacre $M^{r}$ PELsexere ${ }^{1}$ i ces interessants problemes de biogrographie dans son travail sur les Mollusques (p. 33-77).

Ce n'est pas seulement le probleme des rapports entre les leux régions polaires, mais aussi colui des relations de lAntarctile avee les continents voisins, Amérique du Sud ou Australie, que $\mathrm{l}=$ Pestexern precise dans son travail sur les Mollusques. Mais, a ret igard, le mémoire de $\|^{\circ}$ Doboo sur les Poissons est bien plus important encore. Des poistons proptement antartiques, cest-it-dire vivant a l’intrideur du cercle polates, un serb, fait extraordinaire! avat ete decrit et ligurs, mais non recueilli, arant l'expédi-

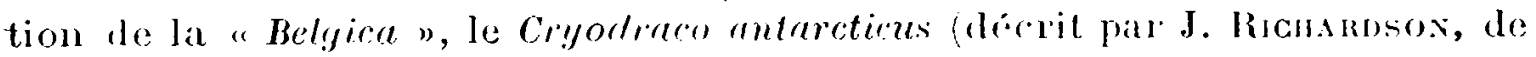
l'expédition James C. Ross). I a "Belgirn" a rapporté les premiers poissons abystaux : le Cryodraco, plus quatre especes nouvelles. Lal "Southern Cross" a, de son coté, recueilli les premieres cspieces priagiques et littorales.

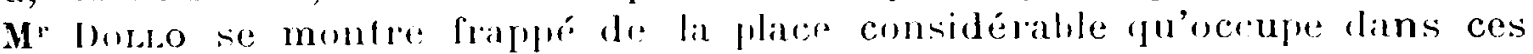
documents nouveaux, ainsi d'ailleurs que dins la faune des poissons subanfaretiplues, la famille des Nothoteneidee, de nature essentirllement marine et plus patriculierement litforale. Pour en expliquer la repartition, il se trouve amene a croire quelle serait un des dermiers restes de la finune australe miociene, et quelle aurat peuple les rivages d'un continent aujourdhui démantelé, réunissant la Palagonie a l'Australie, it travers l'Antarctide. Il adopte le tracé de cette Antarctide Tertiaire tel que l'a conjecturé récenment II. F. Osbon, professeur i l'Université de New-York. I.es Nothoteniedae, lors du morcellement el de la régression de ce continent, seraient en partio restés autour des îles résultant de sa fragmentation el aussi autour de la pointe inérionale de l'Amérique du s; d'autres, s'adaptant à des conditions de vie nouvelles, auraient peuplé les rives de l'Antaretide actuelle, le Platean continental antaretique, et mime de plus grandes profondeurs. On voit que la réunion de l'Australi. ef de PAmérigue du Sud, postulée depuis longtemps, répond a des rraisemblances de plus en plus strieuses.

Pour les animax supérieurs, Phoques, liseaux el Cólacés, les lalents d'observation de $M^{\mathrm{r}}$ Racovita se sont brillaminent donné carrière. Pour les Phoques, il a rapporté des spécimens qui ont permis à Mr Barnett Il I vilton

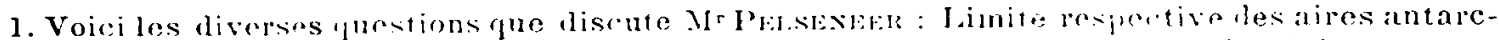
tique et subantaretique (il la tixe aux aborls de 50" de latitule). Subdrision de la bigion antaretique. Relations an irennes des districts subantaretiques contro eux. Comparaison de la faune antarctique arec la faune alyssale. Comparaison de la fano polate anstale avec la taune polaire boreale. 
de determiner enfin les caractères les quatre espices de lhoques qui peuplent l'Antaretique; il a prowve l'inanite des espérances de ceux qui attendaient encore la découverte de nouvelles et étranges formes de Mammifères dans ce monde mystérieux. 1 propos des cétacés on peut dire que Mr Ricovirza, sans avoir pu rapporter un seul spécimen, au sens matériel du mol, a renouvelé la cuestion. La "Belgica" n'a pas vu de baleines franches; elle a surtont observé dans la banquise des exemples isolés de Balenoptera borealis et dHyperoodon el dans les chenaux du détroit de la Belgica de grandes troupes de llégaptires et parfois le gigantesque Balenoplera musculus (2:3 à $30 \mathrm{~m}$. de long). Mr Racoviry décrit avec un intérêt extraordinaire les mours si mal connues de ces inormes animaux, leur facon de sonder, de souffler; les profondeurs auxquelles ils plongent vraisemblablement (selon lui, elles ne peuvent quire depasser une rentaine de metres): surtout il examine lous les renseignements, le plus souvent très vagues, que les explorateurs antérieurs ont lournis sur les cétacés et il s'efforce en terminant de donner un aperru de la Chorologie des Cétacés, c'est-à-dire de leur mode d'habitat et d'existence, de leur nourriture et de la classification qui en résulte, entin des centres probables de formation des deux principaux genres : les Mystacocites et les Denticites.

Les études sur les Oiseaux et sur les lhoques n’ont pas encore paru; on a cependant le droit d'être déji alliché par les observations si pinétrantes fue $\mathbf{M}^{\mathbf{r}}$ Racovitza a publiées dans le Bulletin de la société Royale Belge de géographie en 1900 (Annales de Giographie, Xe Biblio!fraphie 1900, 110906).

Bien que les études sur les glaces antarctiques, sur la geologie et la geographie physique des terres antarctiques, etc., soient également encore à publier, on voit par ce qui a déja paru, toute la portée scientifique de ce voyage, qui avait commencé dans des conditions si modestes, et qui se revele de plus en plus l'une des explorations fondamentales du monde antarctique.

Malrue Zimueraanis.

EXAMENS DE (AEOGRAPHIE 190/

\section{Liste des Mémoires de géographie qui ont valu le Diplôme d'études supérieures d'histoire et de géographie à leurs auteurs pendant l'année 1904'.}

Paris. École Normale Supérieure. - Rext Ml'sset, deographic du Perche. Juillet 190 '.

Faculté des Lettres. - Macmes AtaAx, Le Chene-liegre dins lafrique du Nord. Juillet 190 '.

E. Lexorssox, La Campigne de Caten. Juillet 1904.

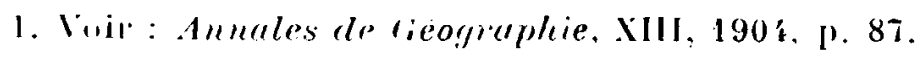

